\title{
Efficacy of a Virtual Environment for Training Ball Passing Skills in Rugby
}

\author{
Helen C. Miles ${ }^{1}$, Serban R. Pop, Simon J. Watt, Gavin P. Lawrence, Nigel W. \\ John, Vincent Perrot ${ }^{2}$, Pierre Mallet, Daniel R. Mestre, and Kenton Morgan ${ }^{3}$ \\ 1 Bangor University, Bangor, United Kingdom, \\ h.c.miles@bangor .ac.uk \\ 2 Aix-Marseille Université, CNRS, ISM UMR 7287 13288, Marseille cedex 09, France \\ daniel.mestre@univ-amu.fr \\ 3 Rygbi Innovations Cyf., United Kingdom
}

\begin{abstract}
We have designed a configurable virtual environment to train rugby ball passing skills. Seeking to validate the system's ability to correctly aid training, two experiments were performed. Ten participants took part in ball passing activities, which were used to compare the combinations of different user positions relative to the physical screen, the use of stereoscopic presentation and the use of a floor screen to extend the field of view of the virtual scene. Conversely to what was expected, the results indicate that the participants did not respond well to simulated target distances, and only the users physical distance from the screen had an effect on the distance thrown.
\end{abstract}

Keywords: distance perception, stereoscopy, rugby

\section{Introduction}

There is a great deal of interest in training sporting skills using virtual environments (VEs), for example handball [1], [2], tennis [3], [4], [5], golf [6], [7], [8], baseball [9], [10], [11], cycling [12] and swimming [13]; and discussion concerning the validity of VEs as a training tool for a wide array of different activities [14], [15], [16]. The discussions focus on the possibility of detrimentally affecting the user by teaching a new skill incorrectly or spoiling a skill the user already possesses by providing a training platform that lacks vital cues or provides superficial cues that are not present in the real world. There is no universally accepted method of validating VEs; technologically benchmarking VEs is difficult when each can be quite unique in their software and hardware configuration. Despite this, however, there are a growing number of examples of VEs (and simulators [17]) being regularly used as part of real training regimes in professional sports [18]. Such VEs must therefore be carefully designed with consideration to affordances and feedback, ensuring that any limitations or assistance provided by the VE do not leave gaps in the users learning or dependencies on certain information [19]. 
We have designed a configurable VE to assist in the training of rugby skills using rugby ball passing as the test case. A focus on passing skills has been made because it is a fundamental core skill in rugby; previous work has been undertaken in lineout [20], [21], [22] and attack interception [23], [24], [25], [26] skills training. The experiments detailed in this paper investigate the technology that was initially chosen for the proposed system, with a focus on the ability to perceive virtual distances correctly within the VE.

\section{Related Work}

For sport-based VEs, it is important to constrain the user as little as possible and allow the freedom of movement that they would normally experience when playing the sport. Data projection to a large screen is the most common approach, e.g. [3], [5]. A head-mounted display (HMD) is likely to interfere with the physical activity of the user, as many require large cables trailing from the headset to transfer the visual data. They also prevent the user from seeing their own hands, requiring the use of an avatar; this in turn can lead to issues with latency.

Many systems also employ stereoscopic technology to augment the sense of depth in the virtual scene: shutter glasses were the most popular type of stereo technology over anaglyphic and polarisation glasses [1], [27]. However, the potential detriment of using stereo technology has not been discussed.

The above issues are discussed in more detail in [28]. This review covers both the state-of-the-art technology currently used to build a VE and a number of other issues involving the training of a user in a task involving ball sports. The investigation of these issues has provided the motivation and a knowledge base on which to build the VE for Rugby Skills Training (VERST). This paper describes the VERST system and presents the results of initial experiments using a rugby ball passing task. We describe the development of a physics engine for a rugby ball in flight, and an investigation of the effect of stereoscopic technology and the physical screen configuration on the user's ability to perceive virtual distances correctly.

\section{Building a Virtual Environment for Rugby Skills Training}

The VE, which can be seen in Fig. 1 and Fig. 3, placed the subject in the centre of the field of play in a model of the Millennium Stadium in Cardiff, Wales. Circular archery-style targets of fixed size (1m diameter) were presented to the user, with the centre of the target $1 \mathrm{~m}$ above the ground. The user holds a real tracked rugby ball (which is tethered to the ceiling or attached to the wrist of the player to prevent any damage to the equipment), and must throw the ball to

hit the targets. The data from the tracked ball is input into the physics engine to produce a predicted flight path, shown with a virtual ball. 
Development of the VERST software began with implementing a physics engine to handle the virtual flight path of the ball. It employs a simple ballistic model based on work by Bray and Kerwin [29] on football free kicks, and by Vance et al [30] on the aerodynamics of a rugby ball in flight, which provided drag and lift coeffients. The solution to the flight path of the rugby ball can then be estimated in real-time using the Runge-Kutta 4th Order method. The required input data for this model is the last position vector at which the ball was recorded for continuity between the real and computed movement, and a Cartesian velocity vector based on an average of several velocity estimates taken as the ball was tracked.

To test the accuracy of the physics model, a series of real ball throws (passes) were captured in a motion capture laboratory at Bangor University. The laboratory was equipped with 12 Vicon cameras covering an area of approximately $5 \times 5 \mathrm{~m}$ in the centre of the room, so allowing for only relatively short throws. The cameras were able to capture a full throw with sufficient accuracy and precision, at a rate of $250 \mathrm{~Hz}$. Once the data had been collected, the throws were compared with a Matlab implementation of the physics model. This highlighted the necessity of controlling the capture window to ensure the correct section of the real ball's flight is captured. This lead us to design the ball capturing system to be a small capture window placed approximately $50 \mathrm{~cm}$ in front of the user; with the participant in a fixed position, which would allow only the capture of the ball after being released from the participant's hands.

To begin evaluating the VERST system, an experiment was designed to examine the effects of display technology on the task of hitting a target with a rugby ball.

\subsection{Issues in Depth Perception}

In a virtual training exercise involving targets at different distances, it is vital that depth perception in the scene be as accurate as possible. Using stereoscopic technology to augment the sense of depth in VERST is therefore an aspect that we want to investigate. Stereo displays have been shown to provide a greater sense of presence in the environment [31] and provide a more accurate sense of object size [32]. However, they can potentially also cause a range of other issues, including discomfort (see [33]). Cues to the target distance were also available from monocular depth cues, principally the angular size of the targets at the eye, and height in the visual field.

Depth compression within VEs using both HMDs [34], [35] and large screen displays [36], [37] has also been previously reported but is not well-understood. Standard methods of evaluating the perception of distance in VEs are verbal judgments [36], [38] and blind walking [39], [40]; blind throwing [41] has also been used. Sahm et al [41] found a distance underestimation of $30 \%$ between distances of 3-6m using a HMD, while Piryankova et al [37] found a distance underestimation of approximately $25 \%$ for an experiment using a large screen immersive display. See the review by Renner et al [42] for an overview. 


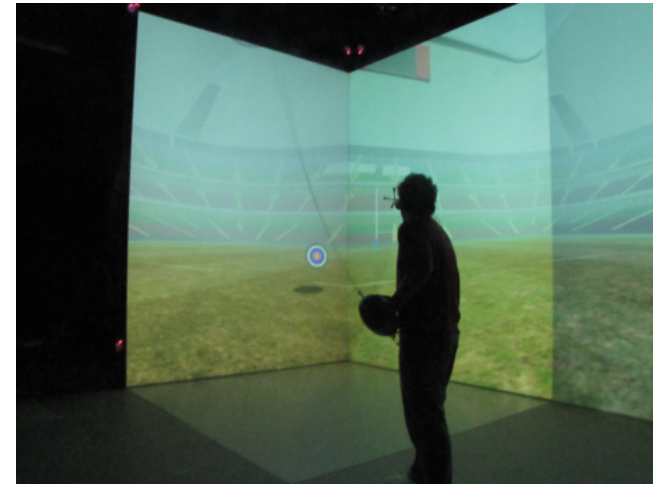

(a)

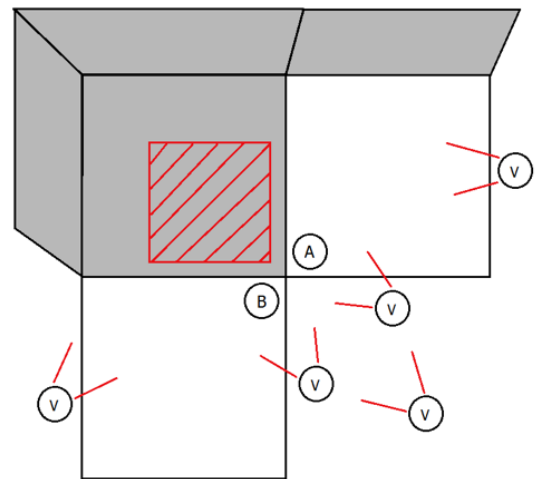

(b)

Fig. 1: (1a) The VERST system during experiment 1. (1b) A top-down view of the screen configuration, the darker sections highlight the screens, the 'A' and ' $\mathrm{B}$ ' markers show the two positions the participant stands during the experiment. The ' $\mathrm{V}$ ' markers give the position of the Vicon cameras and the hatched area indicates the approximate capture area.

\section{An Initial Evaluation of the Efficacy of VERST}

Because of the uncertainty of the effects of stereoscopic technology, and display technology, on the ability of the user to perform our ball passing task, experiment 1 on VERST was to evaluate the suitability of the technology. The performance of the physics engine was also scrutinized, as the results of the trials rely heavily on an accurate representation of the movement of the ball. The key factors to be investigated in this experiment were: (1) stereoscopic versus non-stereoscopic presentation, (2) the use of a floor screen to cover more of the user's field-ofview, and (3) the position of the physical display, where the surface structure is specified by focus cues and a visible outline.

Participants could not see the virtual trajectory of the ball in this experiment. There were two reasons for this. Firstly, we did not want them to adjust their technique to any flaws that may be present in the physics model at this time; we must be confident that it is correctly modeling the ball's movement before exposing users to any potential detriment of training to a non-natural system. Secondly, as the experiment is attempting to measure any distortions in the perception of the virtual target, using the ball throw as the dependent measure, subjects must not be allowed to adapt to any distortions; the perceptual system adapts to systematic distortions quickly, and so allowing feedback would result in excellent performance regardless of any depth distortion problems, thereby preventing measurement of any distortions.

We hypothesize that the participants should respond more accurately to distance in the trials where the floor panel increases the coverage of the participant's field-of-view and stereoscopic cues are available (configurations 1 and 5, see ta- 
ble 1). In the trials from position $B$ the larger right wall screen will also increase the coverage of the participant's field-of-view; this would lead us to expect the best results from trials in configuration 5 . We also expect to see some depth compression in accordance with the previously described literature.

\subsection{Participants}

Four male participants aged 21, 25, 26 and 38 years took part in this preliminary study. The participants had each being playing rugby for a minimum of 10 years for local teams, and played in a variety of team positions. All participants were right-handed and reported having normal, unimpaired vision, or wore their typical corrective lenses (glasses or contact lenses). Participants' stereo vision was tested using the Randot SO-002 to verify each had stereoacuity in the normal range.

Each was observed in a real-world throwing task for a distance of $9 \mathrm{~m}$ to verify their ability to perform passes accurately in the correct distance range. The task was performed indoors to ensure there were no external wind forces affecting the pass, to mirror the conditions of the VE in the experiment.

\subsection{Equipment}

The VERST system was implemented in the 3DVIA Virtools software package and presented in a CAVE-like virtual reality system (Fig 1). The hardware consists of three $3 \times 4 \mathrm{~m}$ rear-projected wall screens and a $3 \times 3 \mathrm{~m}$ floor projected from above. Each screen had a resolution of $1400 \times 1050$ pixels and the screens were seamlessly joined to provide a visually continuous projection surface. The left wall was at 90 degrees from the front wall, while the right wall was "open" (aligned with the front wall), giving an L-shape configuration, to allow the user more space and for efficient placement of the motion tracking system. Stereoscopic projection is achieved using Infitec technology with two DLP projectors per screen.

A tracking system (ArtTrack), using infrared recognition of passive markers placed on the subjects glasses, was used to record the subject's head position and orientation and to update the stereoscopic images relative to the subject's point of view in real time (with a frame rate of $60 \mathrm{~Hz}$ ). The two eyes' images were presented simultaneously and the images were correctly geometrically projected for each eye; prior to the experiment, the interpupillary distance (IPD) of each participant was measured using an Essilor Digital Corneal Reflection Pupilometer (CRP) in order to tailor the separation of the stereoscopic images to each individual. The whole projection system was controlled by a cluster of synchronized PCs.

A real rugby ball was suspended from the ceiling of the building, tracked by five Vicon cameras indicated in Fig. 1b with ' $\mathrm{V}$ ' markers. The participants were positioned at either A or B (marked in Fig. 1b); the white squares in Fig. 1b represent two platforms raised approximately $10 \mathrm{~cm}$ from the ground either side of the CAVE floor screen. The capture area (marked by the red hatched box in 
Fig. 1b) was positioned far enough away from the user that they would have to reach unnaturally to have their hands within it.

\subsection{Experimental Design}

Three variables were used to determine a testing configuration: (1) position of the participant (position A/B in Fig. 1b); (2) whether stereoscopic presentation was active or not (S/NS); and (3) whether the floor screen was active or not $(\mathrm{F} / \mathrm{NF})$. The three variables produced eight different configurations as shown in table 1.

Virtual targets were considered in a range of 5-15m away from the participant into the virtual scene. For each configuration, the participant was presented with 7 target distances with 5 repetitions of each target distance rotated around the azimuth of the user. This resulted in each participant throwing 280 trials. The eight configurations were randomized across participants to remove any order bias.

Table 1: Configurations as defined by each set of variables

\begin{tabular}{cccc}
\hline $\begin{array}{c}\text { Configuration } \\
\text { Number }\end{array}$ & $\begin{array}{c}\text { Position } \\
\text { A/B }\end{array}$ & $\begin{array}{c}\text { Stereo (S)/ } \\
\text { No Stereo (NS) }\end{array}$ & $\begin{array}{c}\text { Floor }(\mathrm{F}) / \\
\text { No Floor (NF) }\end{array}$ \\
\hline 1 & $\mathrm{~A}$ & $\mathrm{~S}$ & $\mathrm{~F}$ \\
2 & $\mathrm{~A}$ & $\mathrm{~S}$ & $\mathrm{NF}$ \\
3 & $\mathrm{~A}$ & $\mathrm{NS}$ & $\mathrm{F}$ \\
4 & $\mathrm{~A}$ & $\mathrm{NS}$ & $\mathrm{NF}$ \\
5 & $\mathrm{~B}$ & $\mathrm{~S}$ & $\mathrm{~F}$ \\
6 & $\mathrm{~B}$ & $\mathrm{~S}$ & $\mathrm{NF}$ \\
7 & $\mathrm{~B}$ & $\mathrm{NS}$ & $\mathrm{F}$ \\
8 & $\mathrm{~B}$ & $\mathrm{NS}$ & $\mathrm{NF}$ \\
\hline
\end{tabular}

\subsection{Procedure}

Once the experiment began, the program automatically assigned the seven target distances randomly between 5 and $15 \mathrm{~m}$ at random angles rotated around the position of the participant (obtained from the tracked glasses). A beep signified the start of the trial, and the participant had 5 seconds to attempt the throw. This ensured that the participant could not have time to study the target and contemplate the distance, and to encourage the participant to react to his first instincts.

If the user failed to throw the ball in time or the Vicon cameras did not successfully capture the motion of the ball, they were awarded one further attempt at the same trial. Despite there being an existing physics model, the virtual ball 
was not shown to the user so that they could not make any corrections to their method or adjust their attempts to compensate for any failings in the system.

The participant was prevented from knowing whether they had successfully hit any of the targets during or after the experiment. After the participant had completed the experiment, they were asked to provide some verbal feedback on the system.

For each trial, the following data were collected: (1) position of the participant from the tracked glasses; (2) position of the target in the VE; (3) the motion data of the real ball collected by the Vicon system; (4) the data that would have been input for the physics engine (position and velocity vectors).

\subsection{Results}

The straight distance thrown by all four participants, as a function of the virtual target distance, is plotted in Fig. 2a; the five repeat trials to the same target distance are plotted as a single averaged data point.

During the experiment it was observed that participant 4 was constantly throwing the real ball with much less force than the other participants. This participant had been observed successfully throwing $9 \mathrm{~m}$ distances with little effort prior to the experiment, and appeared to be struggling to accept the safety of throwing the real ball so close to the screen. It was found that participant 4 accounted for the lower group of results in Fig. 2a, consistently throwing between 3-5m distances for all target distances (with a standard deviation of less than $0.5 \mathrm{~m}$ for each group of trials). To prevent this lower group from distorting the results of the other three participants, only participants 1-3 will be considered when investigating the distance, as shown in Fig. $2 \mathrm{~b}$.

In Fig. 2c the data from Fig. $2 \mathrm{~b}$ are plotted as a series of linear best-fit lines labeled by configuration. This graph demonstrates much poorer scaling between the virtual distance and the thrown distance than we had expected to see, although the slopes do progress upwards slightly between $6-9 \mathrm{~m}$. Because the participants were throwing at targets suspended $1 \mathrm{~m}$ above the ground rather than a target placed on the ground plane, it is not necessary for the participant to throw the exact distance to hit the target, so throws travelling beyond the target distance for the closer targets may not present a problem. To look at the difference between the variables in greater detail, each of the three will be discussed in turn.

Comparison of the different configurations using ANOVA revealed that there was no significant difference in the distances thrown from the two different positions $(F(1,68)=0.22, p=0.64)$, with stereoscopy active/inactive $(F(1,68)=$ $0.18, p=0.67)$ or the floor screen active/inactive $(F(1,72)=0.14, p=0.71)$.

It was noted that there were a great number of trials where the ball missed the target to the left or right. Exploring this revealed that all participants threw too far to the left in $82 \%$ of the trials from position B, and threw too far to the right in $51 \%$ of the trials from position A (in this instance participants $2-4$ threw $66 \%$ of the trials to the right but participant 1 threw only $10 \%$ of the throws to the right). Exploring the statistics of the misses with ANOVA verified 


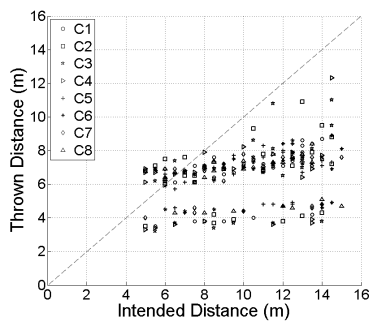

(a)

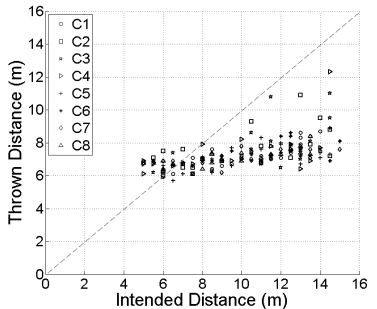

(b)

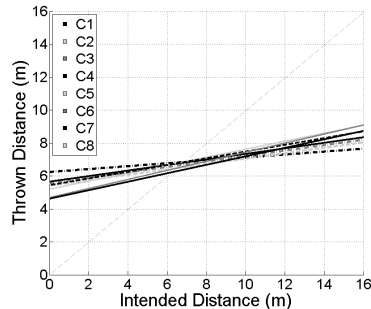

(c)

Fig. 2: Each configuration is plotted in a different shade (c1-8). The dashed line denotes the 1:1 ratio of the two axes. (2a) All participants, the average distance thrown as a function of target distance. (2b) Participants 1-3, the average distance thrown as a function of target distance. (2c) Participants 1-3, the average distance thrown as a function of target distance from $(2 \mathrm{~b})$ presented as a series of best-fit lines.

that there was a significant effect on the left misses from position $\mathrm{B}(F(1,40)=$ $108.3, p=6.04 E-13)$, and right misses from position $\mathrm{A}(F(1,40)=69, p=$ $3.07 E-10)$. Positioning in the CAVE was the only variable influencing this; the number of misses left and right were very evenly spread between the stereo on/off (left: $F(1,40)=0.34, p=0.56$; right: $F(1,40)=0.17, p=0.68)$ and the floor projection on/off (left: $F(1,40)=0.87, p=0.36$; right: $F(1,40)=1.01, p=0.32$ ) variables.

Participant 1 had been in the CAVE previously for other studies; the difference in results, the lower number of misses to the left and right of the target compared to the other three participants is perhaps due to this previous experience. The other three participants had not undertaken any previous experiments at the CAVE used for the experiment, or any other to our knowledge.

Verbal feedback from the participants included two noting that the distances appeared correct to them, and one noting that he used the pitch markings in the virtual scene to aid him. Two of the participants stated that they noticed no difference when the variables were switched, while a third had noticed the floor being changed but not the stereo. Finally, two of the participants commented that it was "strange" and difficult to perform the task while unable to see the virtual ball. None of the participants noted any nausea or discomfort at any point during or after the experiment.

\subsection{Conclusions}

In this preliminary study, it was found that the participants did not respond well to the simulated distances with most throws travelling between $6-9 \mathrm{~m}$ with little regard to the depicted target distance. The use of stereoscopy and the use of a floor screen did not significantly affect the distance thrown by the participant. 
It was noted that the physical position of the participant and the position of the target they were aiming at may have influenced them to over-rotate their throw to the left and right. It is also possible that the participants were unintentionally aiming the throw closer to the diagonal across the floor screen-the longest physical space to throw; for this reason the L-shape configuration of the CAVE may not be appropriate to the task of throwing a rugby ball at a virtual target.

One of the participants was unable to make any throws beyond $5 \mathrm{~m}$, which nullified the training potential of the system for them. It appeared that they were unable to accept the safety of the system, and were fearful of hitting the CAVE walls with the ball. It is conceivable that improving the sense of presence the system provides might assist the participant's performance. This demonstrates that the use of this VE for training will not be suitable for all users, as it is likely that some other users will experience similar problems in accepting the virtual content and ignoring the physical distance.

This experiment showed that, for this VE, users were unable to correctly perceive the distances; further work must be done to understand the reasons for this. The position of the user, the use of stereoscopic presentation and the use of a floor screen to extend the coverage of the user's field of view did not significantly affect the distance estimations made by the participants. Finally, it was found that the shape of the CAVE may not be suitable to this throwing task, as the participants were found to over-rotate their throws.

\section{Further Evaluation of the Depth Perceived in VERST}

The main focus of experiment 2 was to investigate the use of different screen shapes, considering whether this might have affected the results of the throwing task. Participants performed the same task as in the first experiment, using both curved and flat screens. We were given access to a different facility than that used for experiment 1, with both high fidelity curved and flat display environments. However, because the tracking systems available at the new facility only operate at $60 \mathrm{~Hz}$, they would not provide sufficient tracking support for the throwing task. In order to use both the screens, a verbal estimation task was performed with both configurations, and the throwing task with the flat screen only, with the intention of using the verbal results to draw comparisons between the curved and flat screens, and theorise about the performance of the participants in the throwing task using the curved screen. The results from the two experiments are not expected to be perfectly matched for each participant [43], [44], [45], but it is possible that some comparison between the results may be found.

Based on Renner et al's [42] review of distance perception experiments, the distances in the verbal estimation task are expected to be underestimated by approximately $26 \%$.

\subsection{Participants}

Six participants aged 20-54 years took part in experiment 2. For this experiment, the participants were not required to have extensive previous experience 
playing rugby; five of the participants had played only at school or college level, while one had played for a local team. Five of the participants were right-handed, while the sixth was left-handed; all reported normal or corrected-to-normal vision. Participants were all found to have stereoacuity in the normal range using random dot stereograms.

Each was observed in a real-world throwing task to verify their ability to perform passes accurately in the correct distance range. As with experiment 1 , the task was performed indoors to ensure there were no external wind forces affecting the pass, to mirror the conditions of the VE in the experiment.

\subsection{Equipment}

The VERST system was implemented in the Unity 3D and MiddleVR software packages and presented on a flat (shown in Fig. 3a) and a curved power wall (shown in Fig. 3b).

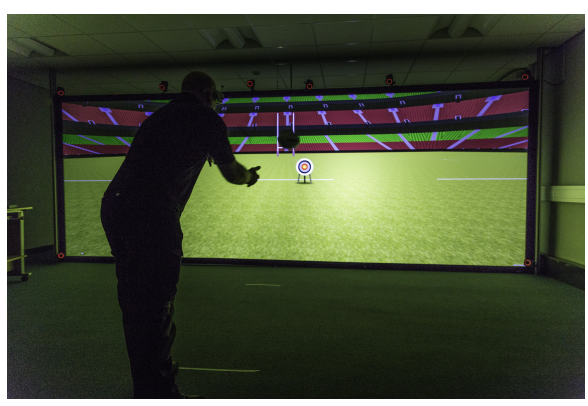

(a)

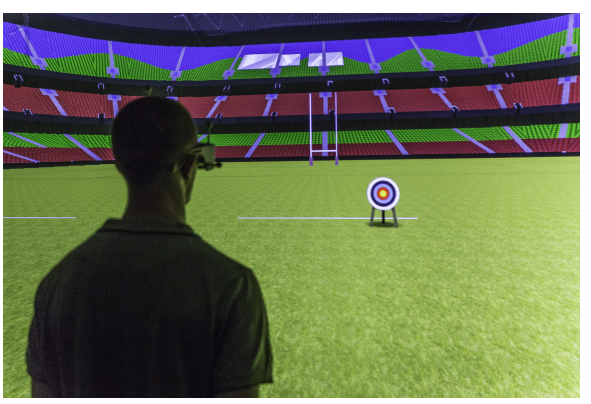

(b)

Fig. 3: Participants during the experiment using the flat (3a) and curved (3b) screens.

The curved screen was $2 \mathrm{~m}$ tall with an arc length of $11 \mathrm{~m}$ (cord length approximately $8 \mathrm{~m}$ ). The screen was rear-projected by 8 separate projectors that ran on either a single unit or a cluster of 8 PCs, depending on the demands of the application. The screen had a resolution of $8500 \times 1920$ pixels. An ART tracking system consisting of 12 cameras was fixed around the edges of the screen; the cameras were able to track multiple objects in front of the screen at $60 \mathrm{~Hz}$.

The flat screen was $6 \mathrm{~m}$ wide and $2.1 \mathrm{~m}$ tall with a resolution of $3387 \times 1200$ pixels from 4 projectors. The laboratory housing the flat screen was equipped with a 12-camera Vicon system that covered the entire floor area in front of the screen by placing the cameras high on the walls all around the room. The cameras were able to capture movement at $250 \mathrm{~Hz}$, making it more than capable of capturing high-speed ball movements.

Both screen configurations had stereoscopic projection, presented through tracked shutter glasses, which allowed the scene to be geometrically projected 
correctly for each user. Before the experiment, each participant's IPD was measured using a basic IPD ruler, and taken into account in the stereo projection.

The change of location for the second experiment meant that the real ball could not be suspended from the ceiling, so a bungee and wrist strap were used for the throwing experiment (see Fig. 3a). The capture area for the ball was determined during each trial as being $1 \mathrm{~m}$ in front of the user (based on their position from the tracked glasses).

\subsection{Experimental Design}

Two variables were used to determine a testing configuration: (1) whether stereoscopic presentation was active (S) or not (NS); and (2) the physical distance of the participant from the screen (Near [1m], Medium [3m] and Far [4m]-the throwing task used only Medium and Far). The two variables produced six different configurations as shown in table 2 .

Virtual targets were considered in a range of 5-10m away from the participant into the virtual scene. For each configuration, the participant was presented with six target distances with three repetitions of each target distance rotated around the azimuth of the user. This resulted in each participant throwing 288 trials. The six configurations were randomized across participants to remove any order bias.

Table 2: Configurations as defined by each set of variables

\begin{tabular}{cccc}
\hline $\begin{array}{c}\text { Configuration No. } \\
\text { (Verbal Task) }\end{array}$ & $\begin{array}{c}\text { Configuration No. } \\
\text { (Throwing Task) }\end{array}$ & $\begin{array}{c}\text { Participant's Distance } \\
\text { from the Screen }\end{array}$ & $\begin{array}{c}\text { Stereo (S)/ } \\
\text { No Stereo (NS) }\end{array}$ \\
\hline 1 & - & Near & $\mathrm{S}$ \\
2 & 1 & Medium & $\mathrm{S}$ \\
3 & 2 & Far & $\mathrm{S}$ \\
4 & - & Near & $\mathrm{NS}$ \\
5 & 3 & Medium & $\mathrm{NS}$ \\
6 & 4 & Far & $\mathrm{NS}$ \\
\hline
\end{tabular}

\subsection{Procedure}

The two screen configurations were alternated for each participant; when the participant was ready they were lead to the first room and performed the verbal task. If they were in the curved screen laboratory, they then moved straight to the flat screen room, but if they were in the flat screen laboratory they remained to perform the throwing task before moving to the curved screen room.

For both tasks, the participant would stand at the instructed position for each configuration of both tasks. A bull's-eye style target would appear in the virtual playing field in front of them; they would have five seconds to react to the 
target by either stating the target distance or throwing the ball at the target. For the throwing task, the participant was prevented from knowing whether they had successfully hit any of the targets during or after the experiment. For the verbal task, the participants were asked to provide an answer in whole metres. The participants were not given any information about the range of target distances, and any comments or questions regarding the distance of the targets or the participant's three positions were neither confirmed nor denied by the experimenter.

For all of the trials in both of the verbal and throwing experiments, the following data were collected: (1) position of the participant from the tracked glasses; (2) position of the target in the virtual space and the distance from the participant; (3) the amount (in degrees) the target was rotated around the participant; (4) the start and completion timestamps for each configuration and the whole experiment. For each of the trials in the verbal experiment, the distance estimate by the participant was noted as the experiment progressed; as the target distances were shuffled prior to each participant performing the experiment, the experimenter only knew the range of possible distances. For each of the trials in the throwing experiment, the following additional data were also collected: (1) the position and velocity vectors that would have been input for the physics engine; (2) the position the ball would land at according to the physics engine. After both the verbal estimation experiment and the throwing experiment, the participants each completed a copy of the Witmer \& Singer Presence Questionnaire [46].

\subsection{Results}

Verbal Experiment First, the individual target distances were analysed (across all participants and configurations, with both the flat and curved screens); the results are plotted in Fig. 4. Medians and inter-quartile ranges are used instead of mean and standard deviation because the distance estimates were made in whole metres.

The data in Fig. 4 shows that the target distances were mostly overestimated, target distances of 5,6,8 and $9 \mathrm{~m}$ only having the lower $25 \%$ of the results underestimated (for 7 and $10 \mathrm{~m}$ target distances, the lower quartile fell $1 \mathrm{~m}$ under the distance they were intended to perceive). The median results for 6 and $7 \mathrm{~m}$ targets are $3 \mathrm{~m}$ above the intended distance, while the 5 and $8-10 \mathrm{~m}$ targets were $2 \mathrm{~m}$ above. The inter-quartile range indicates that $50 \%$ of the estimates were within a range of up to $6 \mathrm{~m}$ across all target distances.

$2 \times 2$ repeated measures ANOVA was conducted on the mean estimated distance values of the verbal task, revealing that the use of stereoscopy did not significantly affect the estimates made using either the curved $(F(1,168)=$ $2.14, p=0.15)$ or flat $(F(1,204)=0.24, p=0.63)$ screens; the combined mean estimated distances were also not significantly affected by the use of stereoscopy $(F(1,348)=0.48, p=0.49)$.

The position of the participant relative to the screen (at Near, Medium and Far distances using both the curved and flat screen) was found to have a sig- 


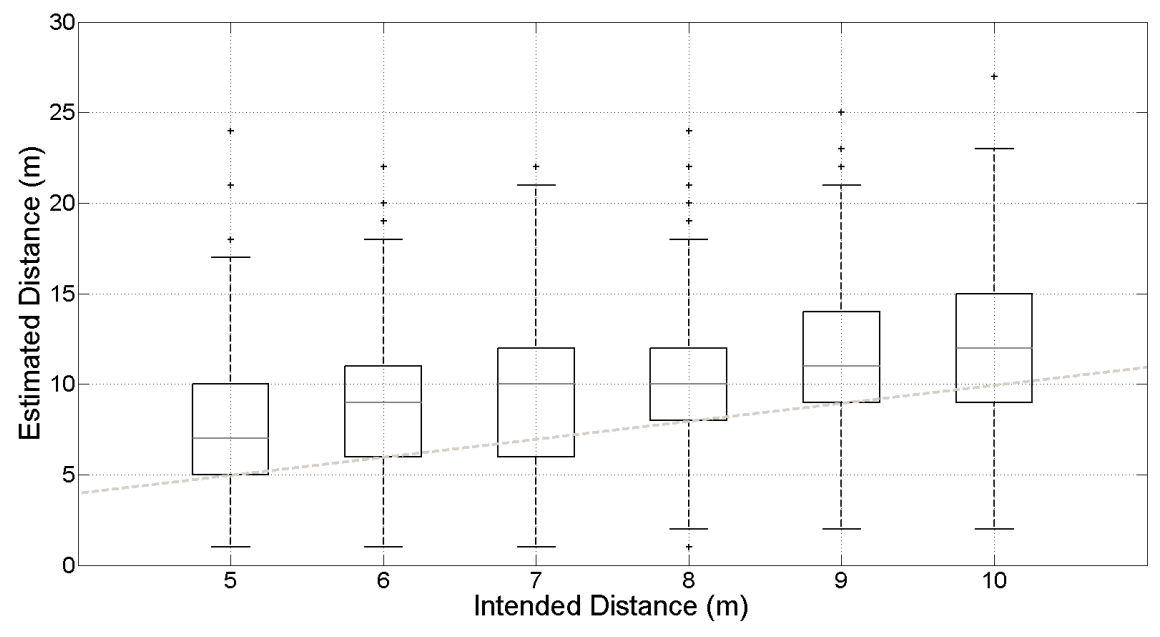

Fig. 4: Results of the verbal task. Intended perceived distance between 5-10m is plotted against the distance estimated by all participants. The dashed line represents the 1:1 ratio of the two axes.

nificant effect $(F(2,342)=28.18, p=0)$ on the estimated distances; target distances were perceived as nearer the closer the participant to the screen. The position of the participant was found to significantly affect the estimated distance when using the curved screen $(F(2,162)=80.25, p=0)$, but not the flat screen $(F(2,198)=1.9, p=0.15)$ (see Fig. 5$)$.

Whether the estimates were made using the curved or flat screen was found to have a significant effect $(F(1,348)=40.4, p=0)$, with mean estimates made using the flat screen consistently overestimated $2-3 \mathrm{~m}$ farther than those made using the curved screen.

Throwing Experiment The mean results of the throwing experiment are plotted in Fig. 6. The results do not appear to be scaled to the intended target distances, with results ranging from $2-30 \mathrm{~m}$ regardless of the target distance.

$2 \times 2$ repeated measures ANOVA was conducted on the mean thrown distance values of the throwing task, showing no significant effect from the use of stereoscopy/no stereoscopy $(F(1,132)=0.4, p=0.53)$ or the distance of the participant from the screen (Medium and Far distances away, $F(1,132)=2.18, p=$ $0.14)$

Presence Questionnaire The Presence Questionnaire was used to assist in gaining an understanding of the participants' attitudes towards different aspects of the VERST system. Comprising of 24 questions, it rates the (1) realism; (2) the possibility to act in the virtual world; (3) the quality of the interface; (4) 


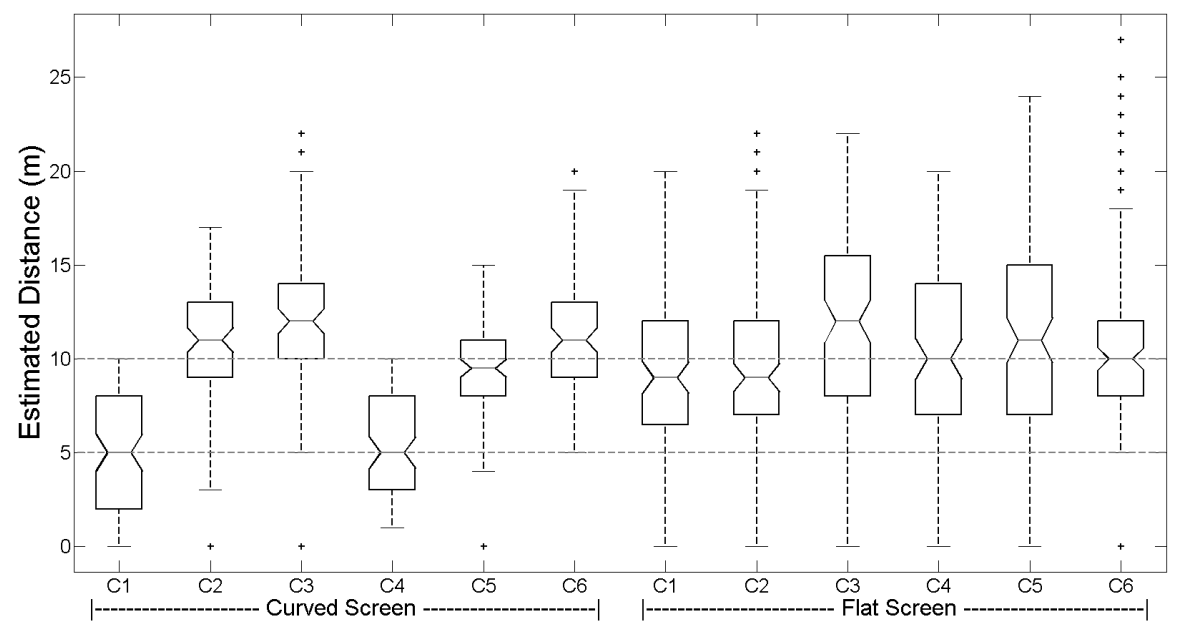

Fig. 5: Results of the verbal task separated into the estimations made under different configurations (see table 2) using the curved and flat screens. The horizontal dashed lines mark the range of target distances intended to be perceived (5-10m).

the possibility to examine the virtual world; (5) a participant's self-evaluation of their performance inside the VE; (6) sounds used in the VE (not used in VERST); and (7) haptics used in the VE.

The results of the Presence Questionnaire are summarised in table 3. No group had significantly different results to the other four, $F(4,25)=0.91, p=$ 0.47, although the Possibility to Examine group has a lower score overall than the other four groups.

Table 3: Presence Questionnaire Group Results

\begin{tabular}{cccc}
\hline Group & $\begin{array}{c}\text { Maximum } \\
\text { Possible Score }\end{array}$ & $\begin{array}{c}\text { VERST Median VERST Inter-Quartile } \\
\text { Range }\end{array}$ \\
\hline Realism & 49 & 37 & 3 \\
Possibility to Act & 28 & 23 & 4 \\
Quality of Interface & 21 & 11 & 4 \\
Possibility to Examine & 21 & 17 & 2 \\
Self-Evaluation of Performance & 14 & 13 & 4 \\
Haptics & 14 & 10 & 1 \\
Total & 133 & 101 & 7 \\
\hline
\end{tabular}




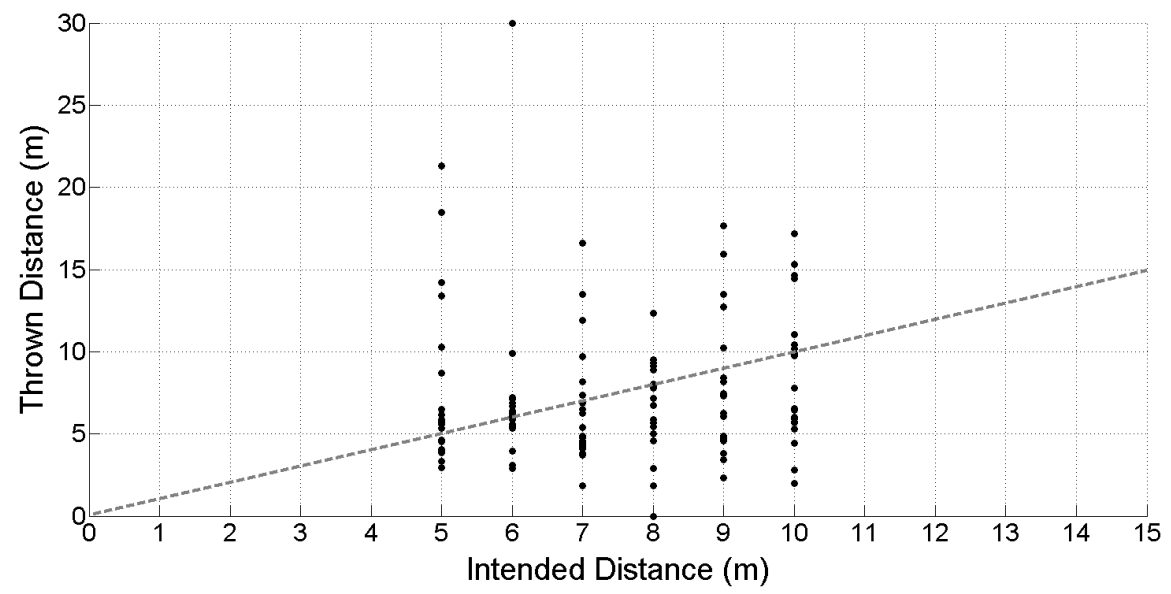

Fig. 6: Distance thrown according to the results of the physics engine plotted against the intended target distance. The dashed line represents the 1:1 ratio of the two axes.

\subsection{Conclusions}

In terms of execution, the second experiment was performed in a similar manner to experiment 1. Differences included the use of the wrist strap rather than a ceiling tether, and a combination of spherical markers attached using hook and loop tape and flat, circular markers for tracking the ball. The wrist strap proved to be difficult to use, like the ceiling tether, resulting in the participants concentrating less on the task and more on the potential of the ball to hit them during the bungee's post-throw reflex. While the spherical markers were held solidly during most of the trials, as the ball hit the ground, the markers would occasionally be knocked off.

The participants responded to the verbal experiment with better scaling to the intended target distances than the throwing experiment. The verbal estimates were generally overestimated by participants, which conflicts with previous similar experiments [42]. The curved and flat screens were found to affect the verbal estimates, with the flat screen estimates tending to be higher than the curved screen. The use of stereoscopy was not found to have a significant effect on the estimations being made in either the verbal or throwing experiments. A significant effect of participant's physical distance from the screen was found for the verbal estimation task, but not the throwing task. The results of the Presence Questionnaire showed that the participants had a positive attitude towards their immersion experience in VERST.

Comparing the results of the verbal and throwing tasks in the second experiment (see Fig. 7) showed an effect of the measurement type, where verbal estimates were tending to be larger than throws. Verbal estimates were scaled 
well to the intended target distance, although they were overestimated, while distances thrown displayed no scaling.

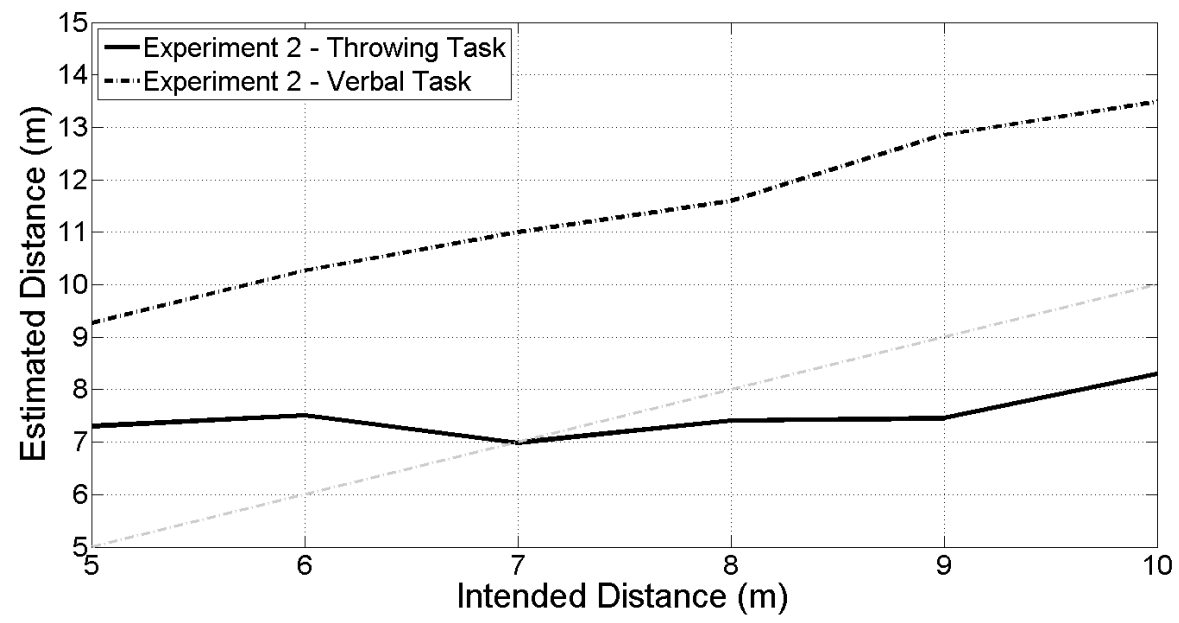

Fig. 7: Estimated distance during the verbal (dashed line) and the throwing (solid line) experiments plotted against the intended distance. The lighter-coloured dashed line represents the 1:1 ratio of the two axes.

\section{Summary and Conclusions}

Two experiments were performed to measure the users' perception of egocentric distance to virtual targets. The first experiment, performed in a CAVE, was based on four participants throwing the tethered ball at 280 targets at $5-15 \mathrm{~m}$ under three experimental variables: the position of the participant relative to the screen, the use of the floor screen and the use of stereoscopy. The second experiment consisted of a throwing task performed using a flat screen, and a verbal estimation task performed using flat and curved screens; experimental variables were the distance of the participant from the screen and the use of stereoscopy. For the second experiment, a smaller range of distances was used: $5-10 \mathrm{~m}$.

The use of stereoscopy was not found to significantly affect the verbal estimations or thrown distances from either experiment; neither was the use of the floor screen in experiment 1 . The position of the participants did affect the distances percieved: in the first experiment, the position of the participant affected the direction of the throw, while for the second experiment, the further the participant from the screen, the further they percieved targets.

The mean throw distance data from both experiments are plotted in Fig. 8. That the participants were able to perceive a scaling in distance during the verbal 
experiment, but not to do the same during the throwing tasks suggests that they may have been uncomfortable with throwing the ball towards the screen.

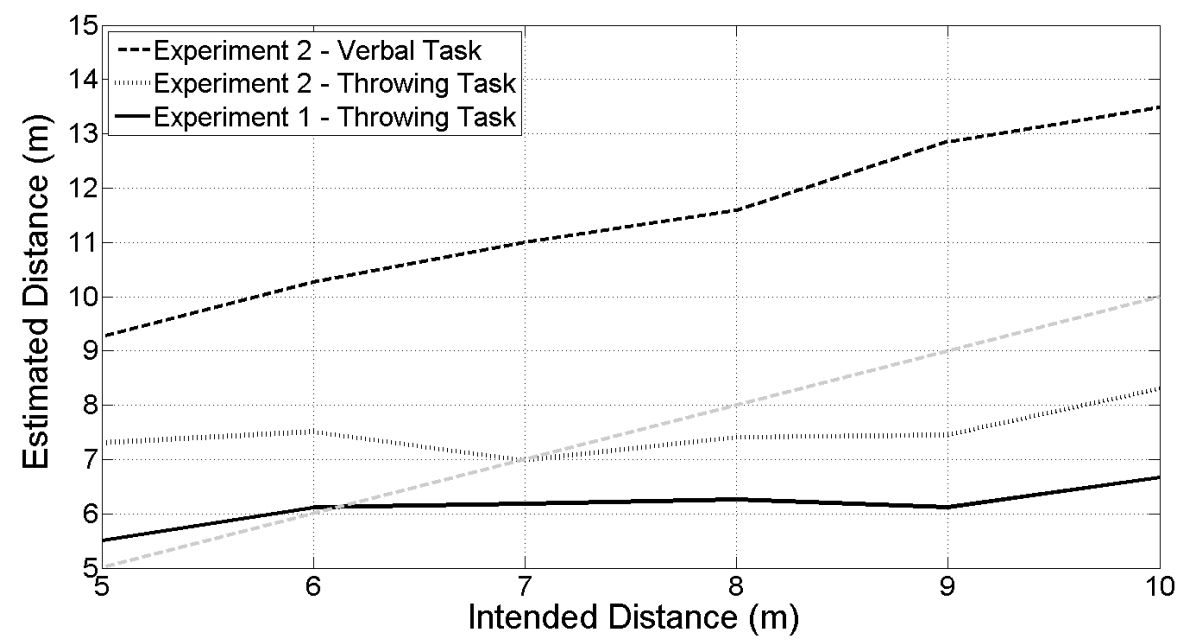

Fig. 8: Comparison of the average estimated distance between the verbal and throwing tasks in the two experiments as a function of the intended distance. The lighter-coloured dashed line represents the 1:1 ratio of the two axes.

A Presence Questionnaire was administered to the participants after the second experiment was performed, which showed that most of the participants had a highly positive attitude towards their immersion experience.

VERST has proved to be a useful platform for investigating the optimal set up of a VE for training ball passing skills in rugby. The system will be improved as a result of the experiments carried out and a validation of skills transfer from the $\mathrm{VE}$ to the rugby pitch will be an important part of our future work. We will also explore training of skills from other ball sports.

\section{Acknowledgments}

The experiment was undertaken at the Centre for Virtual Reality of the Mediterranean (CRVM) at Aix-Marseille University, France, and the Hartree Centre at the Science and Technology Facilities Council, Daresbury, UK. The associates of Bangor University wish to thank both facilities for their hospitality and support during the experiment, particularly Terry Hewitt from the Hartree Centre, and Joss Petit and Chris Greive from OPTIS Northern Europe who supported the experiments at Daresbury.

This project is jointly-funded by a Knowledge Economy Skills Scholarship (KESS), the European Social Fund (ESF), Bangor University and Rygbi Innovations. The research leading to these results has been partially supported 
by the European Community's Research Infrastructure Actiongrant agreement VISIONAIR 262044-under the 7th Framework Programme (FP7/20072013).

\section{References}

1. Bideau, B., Multon, F., Kulpa, R., Fradet, L., Arnaldi, B., Delamarche, P.: Using virtual reality to analyze links between handball thrower kinematics and goalkeepers reactions. Neurosci. Lett., vol. 372, no. 1-2, pp. 119-122, (2004)

2. Bideau, B., Kulpa, R., Menardais, S., Fradet, L., Multon, F., Delamarche, P., Arnaldi, B.: Real handball goalkeeper vs. virtual handball thrower. PresenceTeleop. Virt., vol. 12, no. 4, 411-422 (2003)

3. Xu, S., Song, P., Chin, C.L., Chua, G., Huang, Z., Rahardja, S.: Tennis Space: an interactive and immersive environment for tennis simulation. ICGIP, 652657 (2009)

4. Brunnett, G., Rusdorf, S., Lorenz, M.: V-Pong: an immersive table tennis simulation. IEEE Comp. Graph., vol. 26, no. 4, 10-13 (2006)

5. Li, Y., Shark, L.K., Hobbs, S.J. Ingham, J.: Real-time immersive table tennis game for two players with motion tracking. IEEE-IV, 500-505 (2010)

6. Kelly, P., Healy, A., Moran, K., OConnor, N.E.: A virtual coaching environment for improving golf swing technique. ACM-MM SMVC, 51 (2010)

7. Govil, A., You, S., Neumann, U.: A video-based augmented reality golf simulator. ACM-MM, 489-490 (2000)

8. Ahn, S.H., Kang, K.B., Kim, E.J., Kim, S.J., Lee, J.W., Song, C.G.: Interactive 3D Golf Simulator. Lect. Notes Comput. Sci., (2006)

9. Gray, R.: Behavior of college baseball players in a virtual batting task. J. Exp. Psychol. Human, vol. 28, no. 5, 1131-1148 (2002)

10. Fink, P.W., Foo, P.S., Warren, W.H.: Catching fly balls in virtual reality: a critical test of the outfielder problem. J. Vis., vol. 9(13), no. 14, 1-8, (2009)

11. Syamsuddin, M.R., Kwon, Y.M.: Simulation of baseball pitching and hitting on virtual world. CISIS, 663-667 (2011)

12. Chung, J., Xu, K., Colaco, A., Schmandt, C., Li, V.O.K.: My Second Bike: a tv-enabled social and interactive riding experience. IEEE-CCNC, 1-5 (2010)

13. Fels, S., Kinoshita, Y., Takama, Y., Yohanan, S., Gadd, A., Takahashi, S., Funahashi, K.: Swimming across the Pacific: a VR swimming interface. IEEE Comp. Graph., vol. 25, no. 1, 24-31 (2005)

14. John, N.W.: Design and implementation of medical training simulators. Virtual Reality, vol. 12, no. 4, 269-279 (2008)

15. Hays, R.T., Jacobs, J.W., Prince, C., Salas, E.: Flight simulator training effectiveness: a meta-analysis. Mil. Psychol., vol. 4, no. 2, 63-74 (1992)

16. Crivella, R., Daly, B., Schaaf, R., Ventura, D., Camill, T., Hodgins, J., Pausch, R.: Training for physical tasks in virtual environments: tai chi. IEEE-VR, 87-94 (2003)

17. Thales Group: Thales tackles scrum simulation Thales Group Press Release, 04/06/13. [Online]. Available: http://tinyurl.com/lnbp358. [Accessed: $28 / 05 / 2013]$.

18. Westcott, R.: Trying out Fernando Alonsos Ferrari F1 simulator. BBC News website, 19/04/13. [Online]. Available: http://www.bbc.co.uk/news/technology-22218163. [Accessed: 28/05/2013]. 
19. Ruffaldi, E., Bardy, B., Gopher, D., Bergamasco, M.: Feedback, affordances, and accelerators for training sports in virtual environments. Presence-Teleop. Virt., vol. 20, no. 1, 33-46 (2011)

20. Chong, A.K., Mileburn, P., Newsham West, R., ter Voert, M., Croft, H.: Recent practical applications of close-range photogrammetry for complex motion study. ISPRS J. Photogramm., vol. XXXVII, no. Part B5, 921-926 (2008)

21. Chong, A.K., Croft, H.: A photogrammetric application in virtual sport training. Photogramm. Rec., vol. 24, no. 125, 51-65, (2009)

22. Brook, P., Croft, H., Mann, S.: Laser based line-out simulator. NACCQ, 265 (2007)

23. Bideau, B., Kulpa, R., Vignais, N., Brault, S,. Multon, F., Craig, C.: Virtual reality, a serious game for understanding performance and training players in sport. IEEE Comp. Graph., vol. 30, 14-21 (2010)

24. Brault, S., B. Bideau, B., Kulpa, R., Craig, C.: Detecting deceptive movement in 1 vs. 1 based on global body displacement of a rugby player. IJVR, vol. 8, no. $4,31-36$ (2009)

25. Brault, S., B. Bideau, B., Kulpa, R. How the global body displacement of a rugby player can be used to detect deceptive movement in 1 vs. 1. LavalVirtual, 161-166 (2009)

26. Brault, S., B. Bideau, B., Craig, C., Kulpa, R.: Balancing deceit and disguise: how to successfully fool the defender in a 1 vs. 1 situation in rugby Hum. Movement Sci., vol. 29, no. 3, 412-425 (2010)

27. Vignais, N., Bideau, B., Craig, C., Brault, S., Multon, F., Delamarche, P.: Does the level of graphical detail of a virtual handball thrower influence a goalkeepers motor response? J. Sport Sci. Med., vol. 8, no. 4, 501-508 (2009)

28. Miles, H.C., Pop, S.R., Watt, S.J., Lawrence, G.P., John, N.W.: A review of virtual environments for training in ball sports. Comput. Graph., vol. 36, no. 6, 714-726 (2012)

29. Bray, K., Kerwin, D.G., Modelling the flight of a soccer ball in a direct free kick. J. Sport Sci., vol. 21, no. 2, 75-85 (2003)

30. Vance, A.J., Buick, J.M., Livesey, J.: Aerodynamics of a rugby ball. J. Appl. Mech., vol. 79, no. 2, 021020-5 (2012)

31. Ijsselsteijn, W., Ridder, H., Freeman, J.: Effects of stereoscopic presentation, image motion, and screen size on subjective and objective corroborative measures of presence. Presence-Teleop. Virt., vol. 10, no. 3, 298-311 (2001)

32. Luo, X., Kenyon, R., Kamper, D., Sandin, D., DeFanti, T.: The effects of scene complexity, stereovision, and motion parallax on size constancy in a virtual environment IEEE-VR, 59-66 (2007)

33. Watt, S.J., MacKenzie, K.J.: 3D media and the human visual system. Emerging Technologies for 3D Video: Creation, Coding, Transmission, and Rendering, 349 (2013)

34. Creem-Regehr, S.H., Willemsen, P., Gooch, A.A., Thompson, W.B.: The influence of restricted viewing conditions on egocentric distance perception: implications for real and virtual indoor environments. Perception, vol. 34, no. 2, 191-204 (2005)

35. Willemsen, P., Colton, M., Creem-Regehr, S.H., Thompson, W.B.: The effects of head-mounted display mechanics on distance judgments in virtual environments. APGV, 35-38 (2004)

36. Alexandrova,I., Teneva, P.: Egocentric distance judgments in a large screen display immersive virtual environment. APGV (2010) 
37. Piryankova, I.V., de la Rosa, S., Kloos, U., Blthoff, H.H., Mohler, B.J.: Egocentric distance perception in large screen immersive displays. Displays, vol. 34, no. 2, 153-164 (2013)

38. Interrante, V., Ries, B., Anderson, L.: Distance perception in immersive virtual environments, revisited. IEEE-VR, 3-10 (2006)

39. Thomson, J.A.: Is continuous visual monitoring necessary in visually guided locomotion? J. Exp. Psychol. Human, vol. 9, no. 3, 427-443 (1983)

40. Steinicke, F., Bruder, G., Hinrichs, K.: Transitional environments enhance distance perception in immersive virtual reality systems. APGV, vol. 1, no. 212, 19-26 (2009)

41. Sahm, C.S., Creem-Regehr, S.H.,Thompson, W.B., Willemsen, P.: Throwing versus walking as indicators of distance perception in similar real and virtual environments. ACM Trans. Appl. Percept., vol. 2, no. 1, 35-45 (2005)

42. Renner, R. Velichkovsky, B., Helmert, J.: The perception of egocentric distances in Virtual Environments. ACM Comput. Surv., To Appear (2014)

43. Bridgeman, B., Gemmer, A., Forsman, T., Huemer, V.: Processing spatial information in the sensorimotor branch of the visual system. Vision Res., vol. 40, no. 25, 3539-3552 (2000)

44. Parks, T.E.: Visual-illusion distance paradoxes: a resolution. Atten. Percept. Psychophys., vol. 74, no. 8, 1568-1569 (2012)

45. Goodale, M.A., Milner, A.D.: Separate visual pathways for perception and action. Trends Neurosci., vol. 15, no. 1, 20-25, (1992)

46. Witmer, B.G., Singer, M.J.: Measuring Presence in Virtual Environments: A Presence Questionnaire. Presence-Teleop. Virt., vol. 7, no. 3, 225-240 (1998) 